\title{
Small-scale testing of coal dust explosion propagation and relation to active barrier suppression systems
}

\author{
Catherine Johnson, Barbara Rutter, Christopher Urban \\ Joseph Schott, David Doucet, Chance Moore, Kyle Perry \\ Missouri University of Science and Technology, USA
}

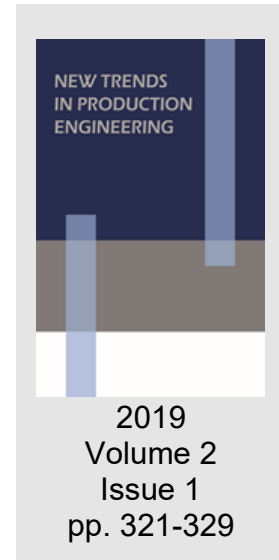

Date of submission to the Editor: 05/2019

Date of acceptance by the Editor: 07/2019

\section{INTRODUCTION}

The risk for underground coal mine explosions has been in existence since coal mining began. The main threat for an underground coal mine explosion is the build-up of methane gas, which can be ignited with a small spark. Once the methane gas is ignited, a shock wave is produced that lifts the coal dust into the air. The fire from the ignited methane gas then traverses throughout all areas of the mine where coal dust was thrown into the air. The shock wave and the resulting fire can result in both casualties and fatalities.

Numerous coal mine disasters have taken place throughout the world. The disasters between 1960s to the present were researched and presented in the following paragraphs and figures. An example of the destructive nature of coal mine explosion in the Upper Big Branch Mine in the United States of America (USA) is shown in Fig. 1 where, the stoppings were ruptured due to the force of the explosion.
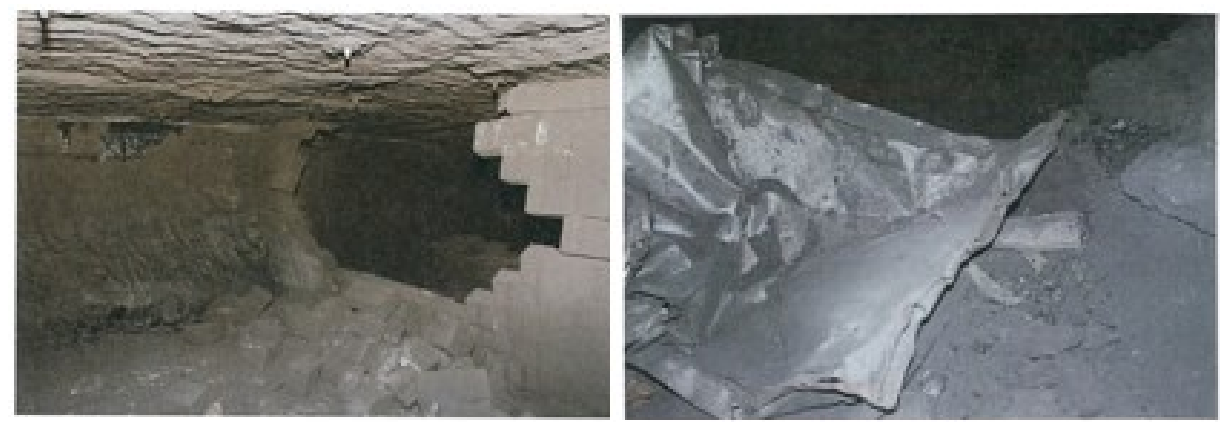

Fig. 1 Photos from Upper Big Branch mining disaster

Source: (McAteer et al., 2011)

The number of coal mine explosions in each country is shown in Fig. 2. China has had the largest number of coal mine explosions. 

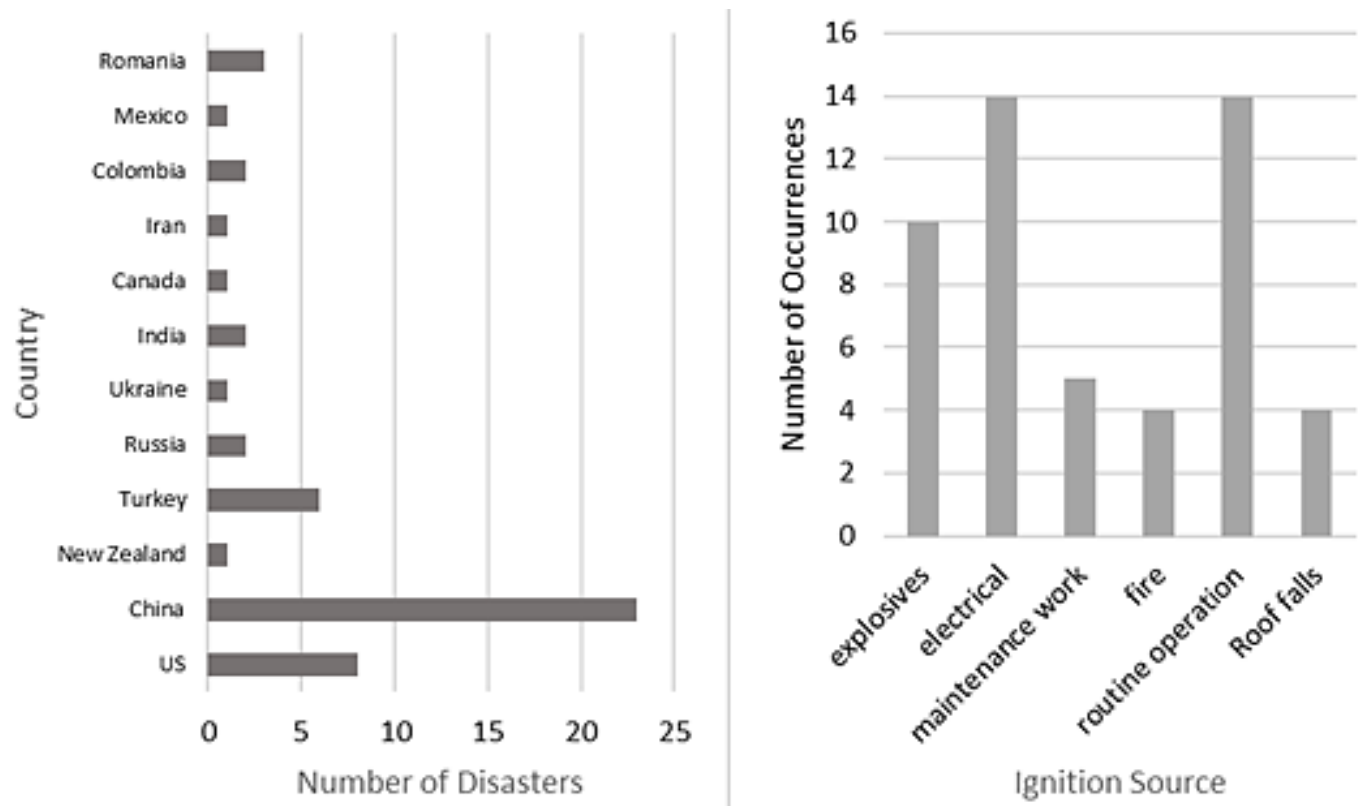

Fig. 2 Number of coal mine explosions in selected countries

Source: (Schafler, 2018)

To combat the expansion of the fireball in a coal mine explosion, suppression systems have been investigated. The most effective method for preventing coal mine explosions is removal of any coal dust accumulations. A common method is coal dust wetting, which prevents the coal dust from becoming airborne, stoichiometrically mixed, and igniting. Another method is mixing of coal dust with inert materials, such as limestone dust, to increase the total incombustibility and prevent combustion. A final line of defense if an explosion were to occur is to use a coal dust explosion suppression method in the form of an explosionactivated barrier with a sufficient amount of inert material. There are two explosion-activated barrier systems, passive and active. The most commonly used barrier systems are passive. One of the most used passive explosionactivated systems is the bag barrier system which places bags of rock dust near the working face of the mine. The bag barrier system is widely used in the United Kingdom, Australia, and South Africa (Schafler, 2018).

The barrier systems have been tested to determine their effectiveness. The Lake Lynn experimental mine of the National Institute for Occupational Safety and Health (NIOSH) was used from 1982-2013 to study mining disasters and possible barrier systems (Sapko et al., 2000; Hill-Davis, 2014). Unlike Lake Lynn, shock tubes and shock tunnels are above ground. The Barbara Mine in Poland has both underground workings and above ground testing sites. All of these experimental sites and equipment are geometrically straight. The research in this paper introduces the use of a small scale model to understand how the shock wave produced from a coal dust explosion traverses a mine with various configurations to the exit should the explosion blow out stoppings and not travel linearly in the path of the explosion barrier system. This paper presents results for 5 different test configurations in a scaled 3 entry longwall gate road system that is common in the USA. Pressure data was recorded along with high 
speed videography to help determine best practices for implementing an active barrier system in the USA.

\section{METHODOLOGY}

Small scale testing was approached by constructing a $1: 10$ scale model of with a typical 3 entry longwall system using $1 / 2$ inch steel and $1 / 2$ inch thick polycarbonate to allow for visualization of the shock wave's movement through the model. Five design configurations were chosen using movable stoppings to see how the shock wave was affected by the different propagation paths and passing through various crosscuts.

All five of the designs used PCB piezoelectric flush mount pressure transducers (model\# 102B, 102B04, and 102B15) placed in the floor and pillars. The sensors were placed in the floor instead of the roof because the polycarbonate top allowed for visualization of the passage of the shock wave. The flush mount sensors were placed at areas where the geometry changed, as shown in Fig. 37 by stars.

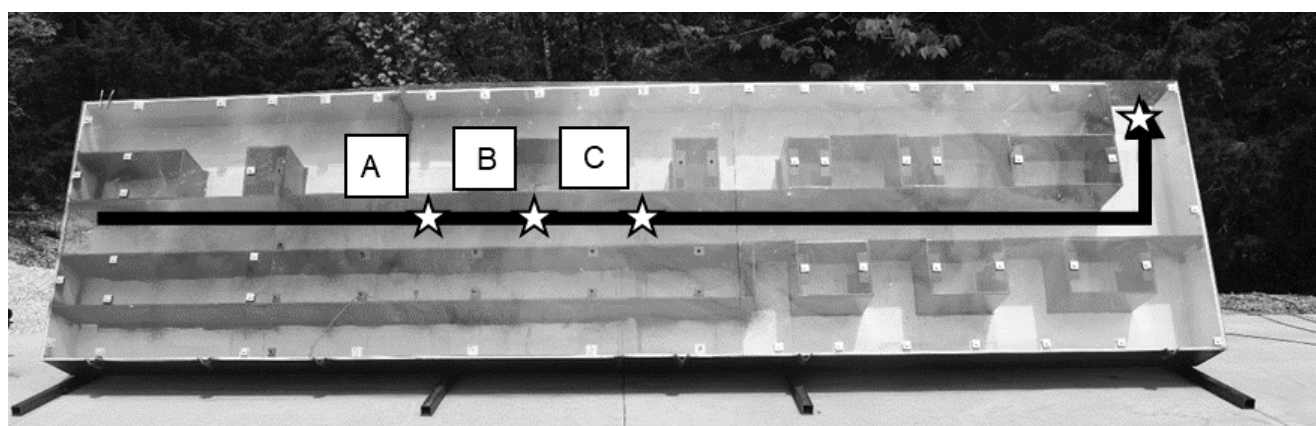

Fig. 3 Straight drift was fitted with sensors in the floor, location indictedby stars, and three pillars labeled A, B, and C. This drift served as the control

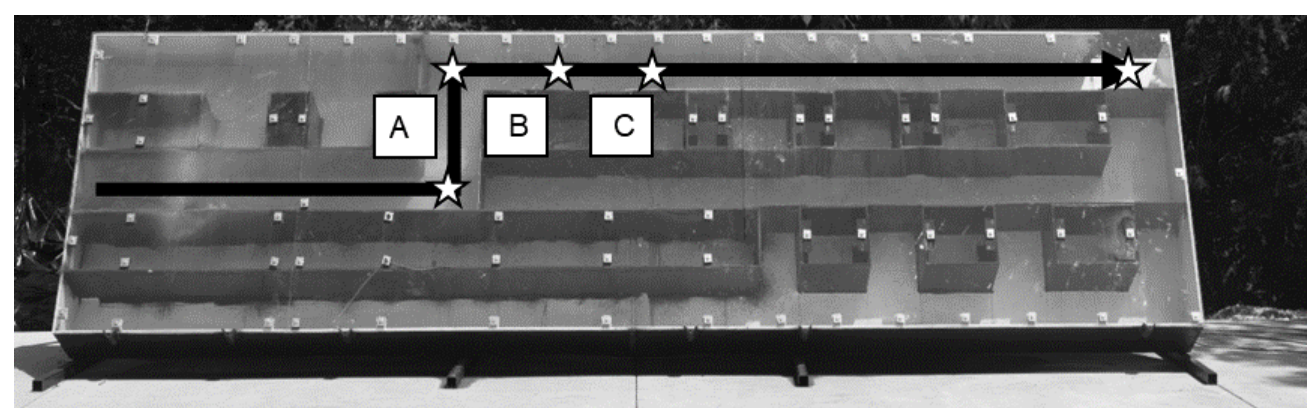

Fig. 4 Single turn drift with sensors in the floor, denoted by stars, and pillars A, B, and C

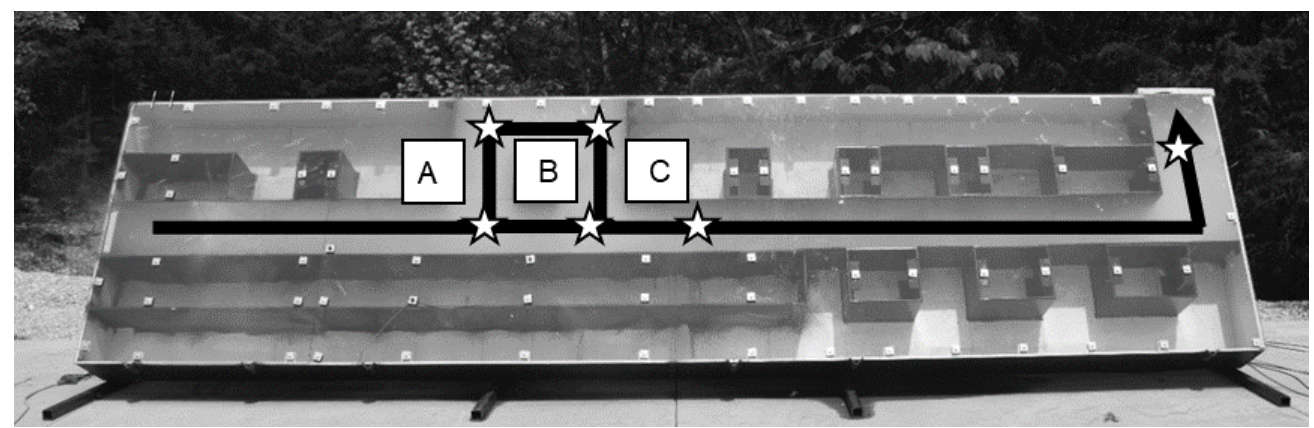

Fig. 5 Around Pillar drift with sensors in the floor, denoted by stars, and pillars A, B, and C 


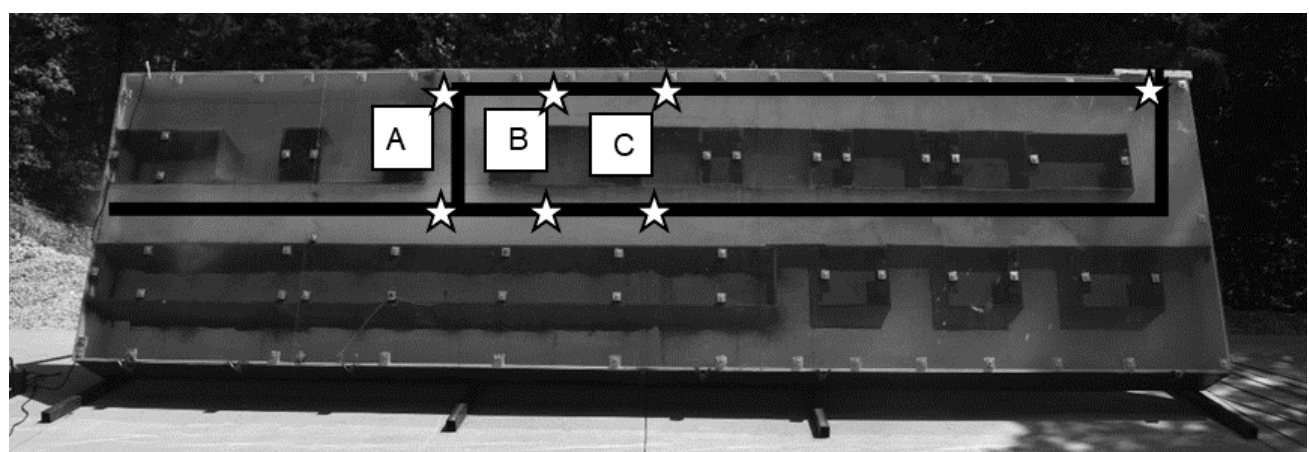

Fig. 6 Split drift with sensors in the floor, denoted by stars, and in pillars A, B, and C

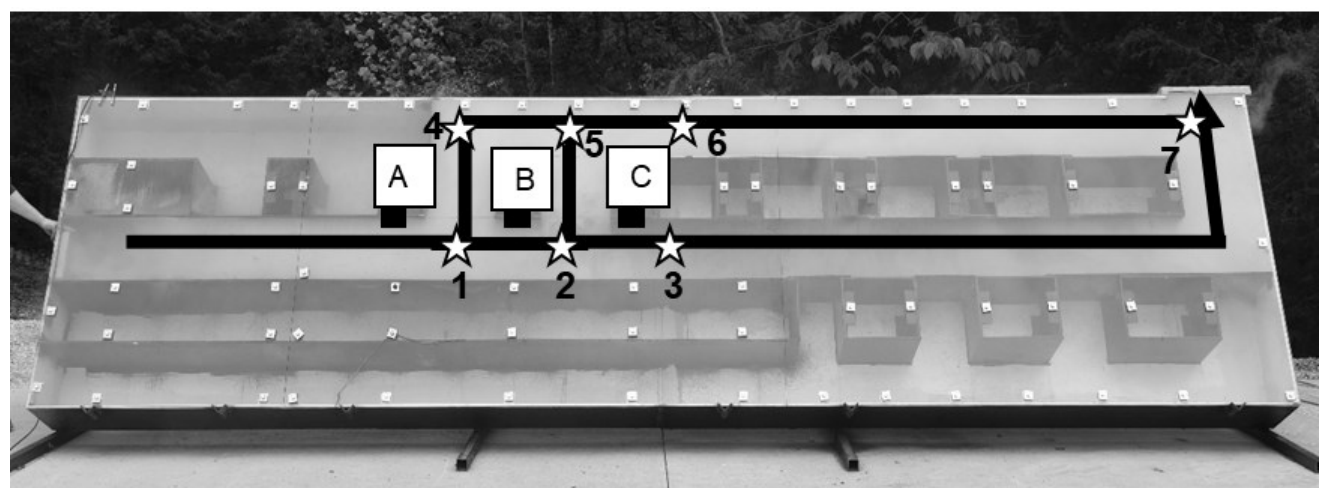

Fig. 7 Split around pillar drift with sensors in the floor, denoted by stars and numbered, and in pillars $A, B$, and $C$

A single pressure transducer was placed in the floor seven inches from the exit. This sensor was used as the baseline for all test designs to allow for easy comparison of the recorded pressure trace. The pressures were recorded by a Hi-Techniques Synergy Data Acquisition System (DAS) that was connected to the pressure sensors with coax cables. A Phantom V2012 high-speed camera was used to record the shock wave's movement throughout the model. The high-speed camera and DAS were triggered with a break-wire placed across the blasting cap, thus ensuring that each iteration was recorded.

\section{RESULTS}

Three tests were conducted for each of the five design configurations. For each test, pressure data and high-speed video was recorded. The model was filled with fog to better visualize the movement of the shock wave on the high-speed footage. The measured pressures, calculated impulses, and rise times were analyzed to determine if volume and velocity of the shock wave had any statistical significance.

\section{DISCUSSION}

Fig. 8 shows a representative pressure trace of a straight drift test for the stared base/sensors labeled in Fig. 7. A single pressure spike is observed at Bases 1, 2, and 3. However, at Base 7 the pressure trace has several small pressure spikes and the much slower rise time. These spikes are the result of the shock wave having lost energy and reflecting off the back of the drift. 


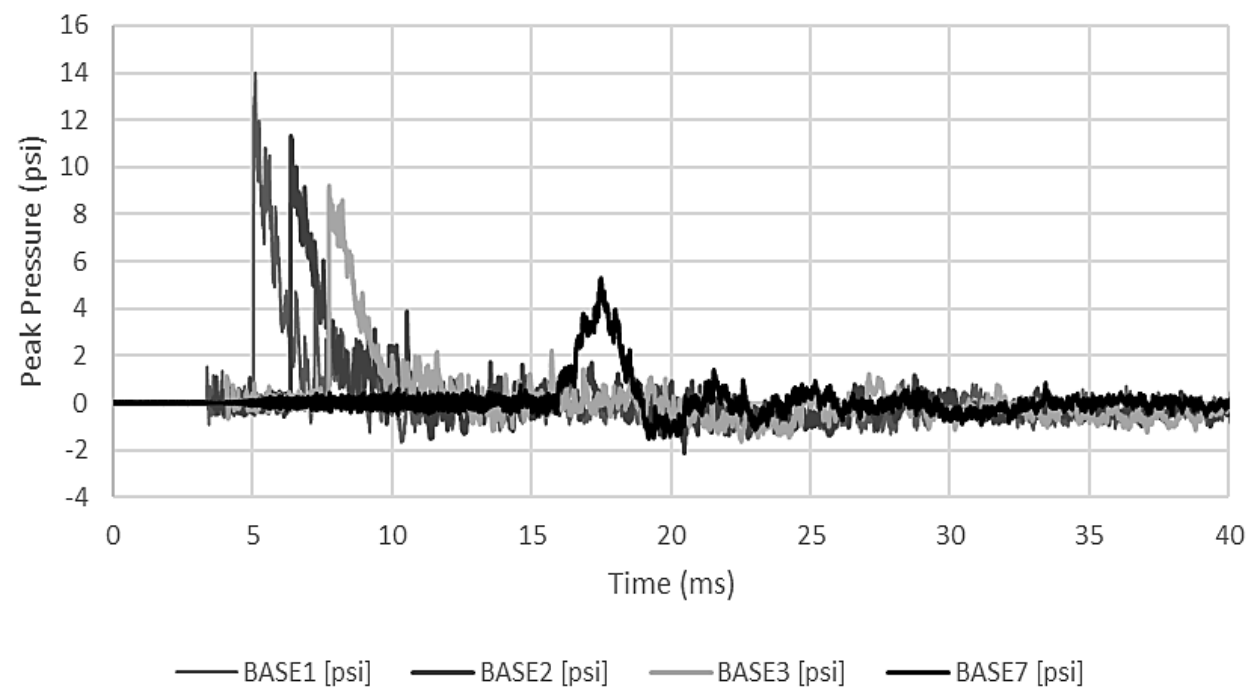

Fig. 8 Pressure versus time for all base sensors in the straight drift

Straight drift series (Fig. 3) was used as the control to compare to the other test pressure traces.

Throughout all the tests the incident impulse was calculated by using the midpoint approximation method, which is the summation of the average pressure over each time step. For the other test designs, numerous reflections were observed, as shown in Fig. 9, and a positive phase duration of $20 \mathrm{~ms}$ was chosen so that all reflections were accounted for. Any negative pressure was not considered for impulse calculation, so all reported impulse values are gross positive phase impulse values.

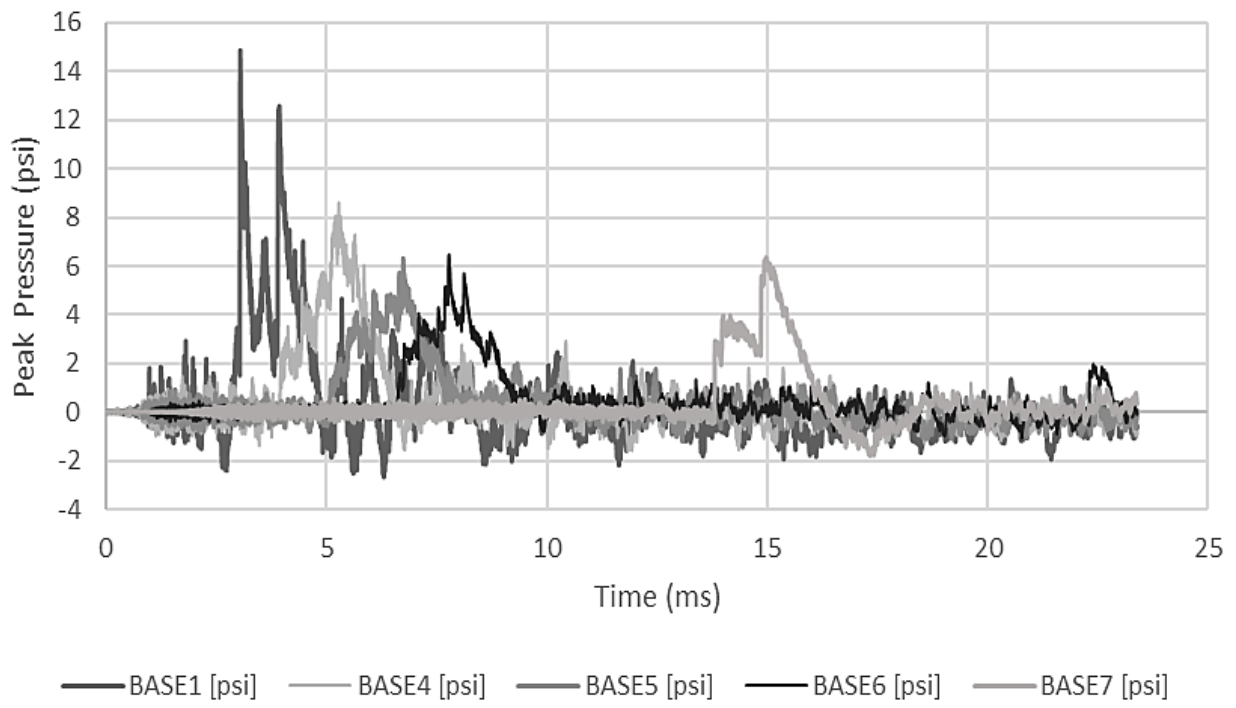

Fig. 9 Pressure versus time for all base sensors in the single turn drift

Fig. 10 and 11 are comparisons of the peak pressure, impulse, and rise time for four sensors: Bases 1, 2, 3, and 7, which are shown as stars in Fig. 7. Thesefour sensors were used in all the test designs except single turn and give the best comparison across all test series. Also included are graphs of the rise time for pillars $A, B$, and $C$, which are shown as rectangles in Fig. 7. Upon analyzing 
both the floor and pillar data, the pillar data was found to be inconsistent, thus roof sensor locations should be used for the trigger sensors.

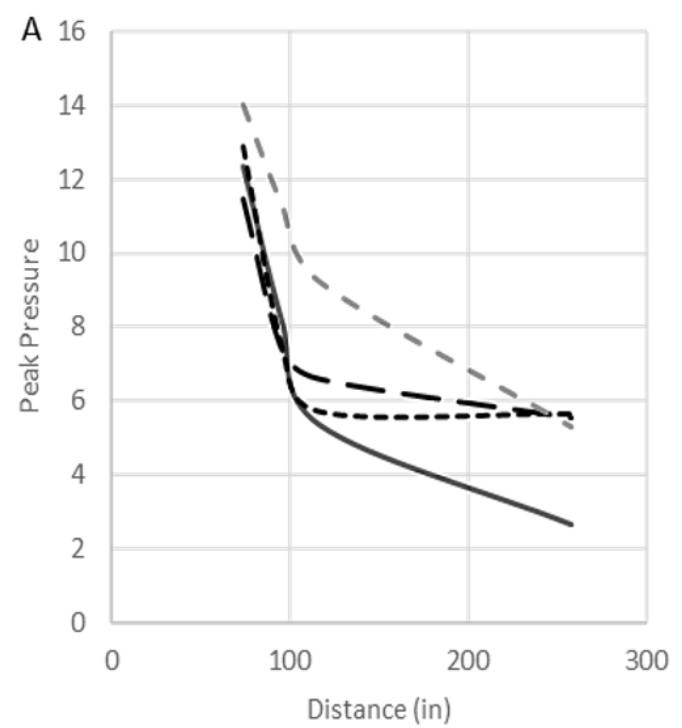

- Around Pillar ------Split Around

$$
\text { - - Split } \quad--- \text { Straight }
$$

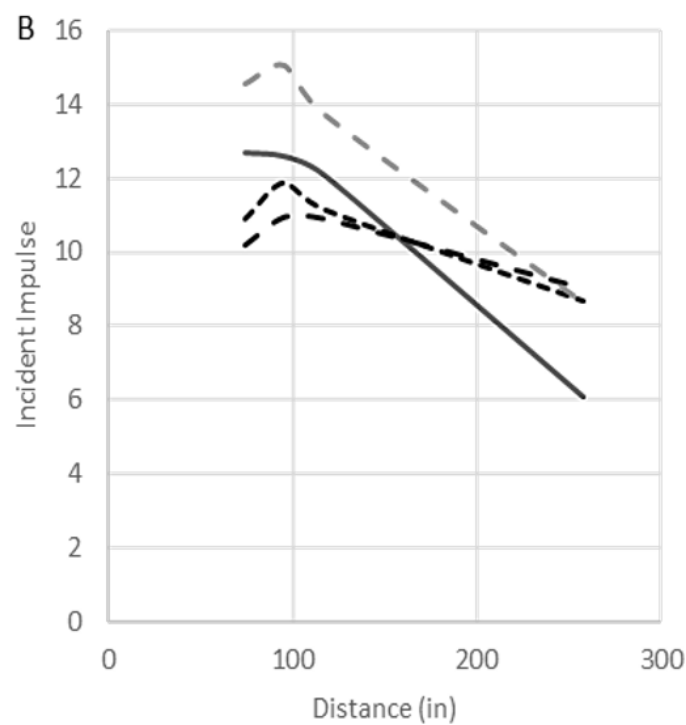

Around Pillar ------Split Around

- - - Split $\quad$ - - - Straight

Fig. 10 A) Peak Pressure versus distance for sensors 1, 2, 3, and 7. The peak pressure decays over distance where the straight drift has the highest peak pressure for all distances.

B ) Impulse versus distance for sensors 1, 2, 3, and 7. The straight and split around drifts have the same initial increase in impulse. The split around and split drifts have a similar decay. The around pillar and straight drifts also have appear to have similar decay rates after Base 2

Fig. 10A shows Peak Pressure versus distance for Bases 1, 2, 3, and 7 while Fig. 10B shows impulse for the same locations. The peak pressure decays over distance, as expected with a single initiation source, but also apparent is the higher pressure for the straight drift across all sensor locations, indicating the volume the waves have to travel will play a large role in the overall waveform. To account for this, the authors initially concluded that all entries and crosscuts need to have sensors placed that advance consistently with production for an active barrier system to trigger. The impulse values also decay as distance increases, as expected, but at a slower rate. Interestingly, the straight and around pillar drifts have the same decay rate for peak pressure and impulse, as the shock wave has to travel along a similar path. The split and split around drifts had nearly identical trends for both peak pressure and impulse is consistent with the shock wave energy being divided between two entries.

Fig. 11A shows rise time versus distance for Bases 1, 2, 3, and 7 while Fig. 11B shows rise time versus distance for Pillars $A, B$, and $C$. The rise times in Fig. $11 \mathrm{~A}$ increase as distance increases from the charge in a linear fashion for all configurations, indicating it will be possible to program sensors to trigger based on a known rise time. As the shock wave travels through the scale model, the shock wave reflects off the walls resulting in the increased rise time. However, this trend is not observed in Fig. 11B. 


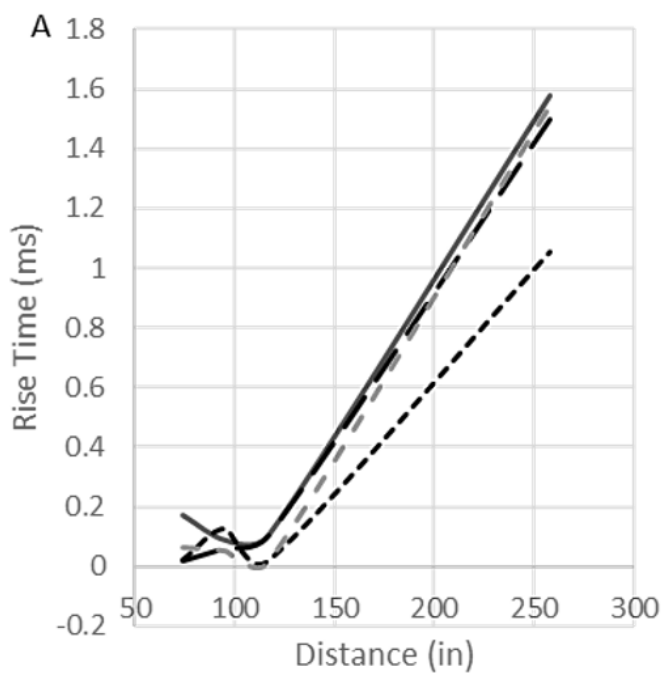

- Around Pillar-----Split Around

- - Split _ - - - Straight

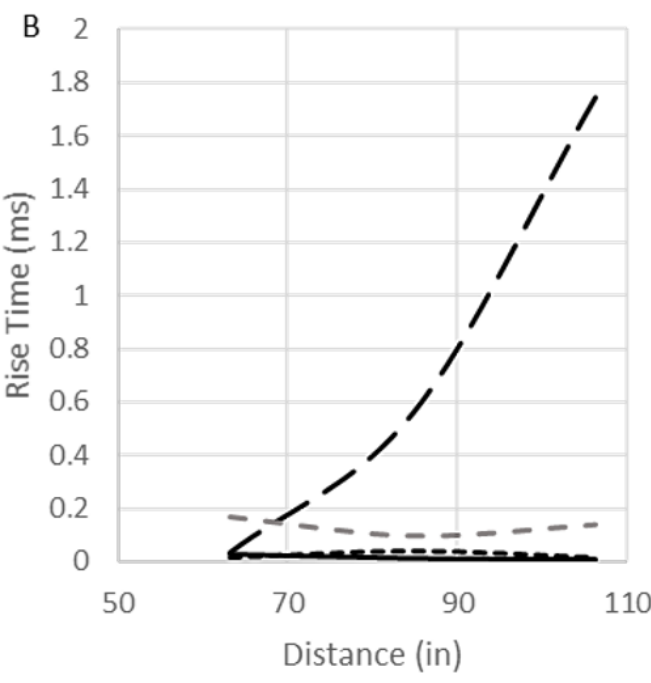

- Around Pillar------Split Around

- - Split $\quad$ - - - Straight

Fig. 11 A) Rise time versus distance for sensors 1, 2, 3, and 7. The rise time increases as distance from the charge increases. B)Rise time versus distance for sensors in Pillars A, B, and $C$, which are the sensors in the path of the straight drift. Straight, around pillar, and split around have similar rise times; whereas, the split has drastically longer rise times

The rise times are relatively stagnant for all tests except for the split drift. The split drift had a long rise time, as expected, due to the shock wave reflecting off the walls and pillars $A$ and $B$. These findings further confirm that sensors need to be placed at every intersection within the mine near the working face and not on the pillars.

Fig. 12 shows the data from Base 1 and Base 7 from each test where Base 1 is on the left and Base 7 is on the right.

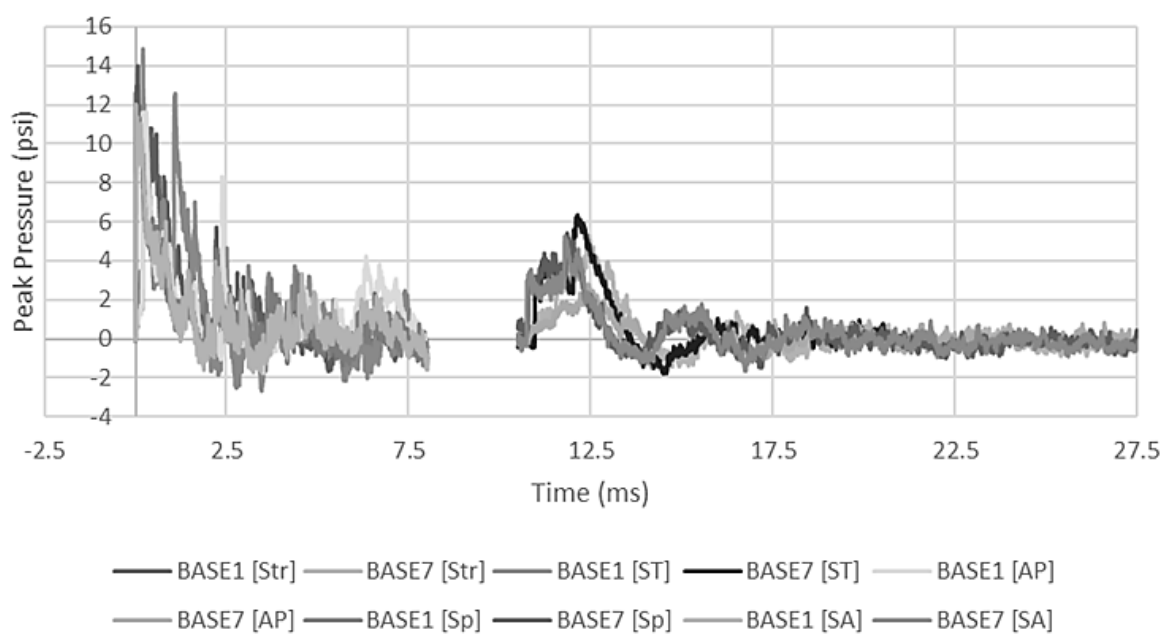

Fig. 12 Pressure versus time for all of Base 1 and Base 7 for each test, where Base 1 is on the left and Base 7 is on the right

One interesting aspect shown in this graph is the impulses at Base 7, the variation is due to a change in rise time and amplitude of the peak pressure dependent of the path the wave takes. For Base 1, the trends are similar for the initial peak. The pressure traces at Base 7 do not have similar trends amongst 
the different test designs. From these observations, the authors further conclude that sensors must be placed at every intersection within the mine as the further you are from the initiation source, triggering an active system based on pressure increase will be inconsistent and possibly result in false triggering or worse, not triggering during an explosion.

\section{CONCLUSION}

Small scale testing was conducted to determine the ideal locations for sensors to be placed in a triggered explosion-activated suppression system. Pressure transducers were placed in the floor at each crosscut and pillar where the geometry changed. Upon analyzing the data, the ideal location for the sensors was at each crosscut positioned on the roof, as the rise times increased uniformly as distance from the explosion increased. The sensors located in the pillars did not have consistent data, thus the authors recommend that the sensors only be placed on the roof at every crosscut as the working face advances.

\section{FUTURE WORK}

Heat, light, and pressure sensors will be placed at the end of the straight drift scale model to see what the best triggering system is. Rock dust, water, and chemical suppressants will be tested to determine the best suppressant. Full scale testing will take place with the best trigger and suppressant at each crosscut to determine if this combination can stop a coal dust explosion from progressing.

\section{ACKNOWLEDGEMENTS}

The authors would like the thank The National Institute for Occupational Safety and Health (NIOSH) for funding this work.

\section{REFERENCES}

Hill-Davis, A. (2014) SECURE NIOSH'S LAKE LYNN EXPERIMENTAL MINE Background. Available at: https://cdn.ymaws.com/www.imana.org/resource/dynamic/blogs/20140612_164243_31683.pdf (Accessed: 20 June 2019).

Mc Ateer, J. D. et al. (2011) Upper Big branch. Available at: http://s3.documentcloud.org/documents/96334/upperbigbranchreport.pdf (Accessed: 20 June 2019).

Sapko, M. J. et al. (2000) 'Experimental mine and laboratory dust explosion research at NIOSH', Journal of Loss Prevention in the Process Industries. Elsevier, 13 (3-5), pp. 229-242. doi: 10.1016/S0950-4230(99)00038-8.

Schafler, J. R. (2018) Management of coal dust explosions in United States' coal mines using bag type passive explosion barriers. Missouri University of Science and Technology. Available at: https://scholarsmine.mst.edu/masters_theses/7782 (Accessed: 19 June 2019). 


\begin{abstract}
.
Coal dust explosions are a lethal threat to anyone working in an underground coal mine. Many coal mining countries including Australia and much of Europe already utilize passive barrier explosion suppressant systems but due to differences in ventilation patterns in the United States, simple passive systems such as the bagged barrier are not as cost effective. Active systems are triggered by properties of an explosion, such as pressure, heat, or light, and release or project a suppressant into the environment to suppress an explosion. To deploy an active system, the best sensor and suppressant release location and spacing must be determined; this must account for total system latency and explosive propagation speed. A 10:1 model of a longwall entry system has been developed to study the pressure wave propagation of coal dust explosions and consequent triggering of different suppressants. The scaled model, with its removable stoppings, allows multiple potential propagation pathways for an explosion to be repeatedly tested, different from typical straight shock tunnel tests. The layout also facilitates the placement of sensors and cameras to fully observe and document the tests. The pressure wave characteristics found at crosscuts and corners will aid in the development of active barrier trigger systems and spacing of suppressant release locations.
\end{abstract}

Keywords: coal dust, explosion, mine, active barrier 